\title{
Desenvolvimento de Arranjo Produtivo Local de Plantas Medicinais e Fitoterápicos em Volta Redonda/RJ
}

\author{
Adriana Passos Oliveira ${ }^{a^{*}}$ e Fabiola Angelita Cezarina Bastos Martins ${ }^{\mathrm{b}}$ \\ ${ }^{a}$ Faculdade de Farmácia, Universidade Federal do Rio de Janeiro. Rio de Janeiro, RJ, Brasil. \\ ${ }^{\mathrm{b}}$ Secretaria Municipal de Saúde de Volta Redonda. Rio de Janeiro, RJ, Brasil
}

Histórico do Artigo Recebido em: 26/10/2017 Aceito em: 28/02/2018

Palavras-chave: Atenção à saúde; plantas medicinais; fitoterápicos; capacitação; assistência farmacêutica.

keywords:

Healthcare; medicinal plants; phytotherapeutic drugs; inservice training; pharmaceutical services.

\begin{abstract}
RESUMO
A Secretaria Municipal de Saúde de Volta Redonda (SMS/VR) desenvolveu ações para implementar a Política Nacional de Prática Integrativas e Complementares (PNPIC) e a Política Nacional de Plantas Medicinais e Fitoterápicos (PNPMF). Nesse contexto, foi publicada a Lei Municipal $\mathrm{n}^{\circ} 4.832$ de 2011, que fortalece a Política Municipal de Práticas Integrativas e Complementares e possibilita a integração com a Política de Assistência Farmacêutica do Município. Para a ampliação do trabalho na área de fitoterápicos, fez-se a estruturação da cadeia produtiva e sua integração na busca do desenvolvimento local, caracterizando o Arranjo Produtivo Local (APL). O trabalho foi desenvolvido de maneira intersetorial e transversal, envolvendo a SMS/VR e as instituições colaboradoras, de acordo com os respectivos eixos A, B, C, D e E. O eixo A abordou o cultivo das espécies medicinais; o B consiste na produção de fitoterápicos por manipulação em Farmácia Viva; o $\mathrm{C}$ compreende a dispensação dos fitoterápicos; o D abrange a capacitação dos profissionais envolvidos e o $\mathrm{E}$ engloba a manutenção das parcerias existentes por intermédio da articulação, da interação e da cooperação, promovendo esforços para o desenvolvimento de medidas voltadas à melhoria da atenção à saúde e a integralidade do cuidado, ao fortalecimento da agricultura familiar, à geração de emprego e renda, à inclusão social. Dessa forma, o APL promoveu o resgate, a valorização, a ampliação e qualificou o uso de plantas medicinais e dos fitoterápicos como elementos estratégicos de saúde.
\end{abstract}

Development of Local Productive Arrangements of Medicinal Plants and Herbal Medicines in Volta Redonda/RJ

\section{ABSTRACT}

The Health Department of Volta Redonda City (SMS/VR) has been developed actions to promote the National Policy on Integrative and Complementary Practices (PNPIC) and the National Policy on Medicinal and Phytotherapy (PNPMF).Thus, a city law number 4.832 of 2011 was published, which strengthens the local Policy on Integrative and Complementary Practices and enables the integration of the local policy with the pharmaceutical services. In order to expand the work of phytotherapeutic drugs, the production process was developed and its integration to promote the local development, characterizing the Local Productive Arrangement (APL). The work was undertaken as an intersectoral and transversal way with SMS/VR and cooperation partners according to axes A, B, C, D and E. The A axis was dealing with medicinal plants cultivation, the $\mathrm{B}$ axis comprises the production of herbal medicines in Farmácia Viva (Green Pharmacy), the $\mathrm{C}$ axis was carried out to distribute phytotherapeutic drugs, the $\mathrm{D}$ axis was conducted to the training of people involved and the $\mathrm{E}$ axis comprises the maintenance of collaborators through articulation, interaction and cooperation to promote efforts to improve the health care and integral care, strengthening family farming and also to offer new jobs and social inclusion. Therefore, the APL promotes the rescue, valorization, extension and qualifies the use of medicinal plants and phytotherapeutic drugs as strategic elements of health.

\section{Introdução}

No Brasil, em 2006, duas políticas foram publicadas para o setor de plantas medicinais e fitoterápicos no Brasil. A primeira foi a Portaria Ministerial MS/GM n ${ }^{\circ} 971$, de 03 de

\footnotetext{
${ }^{*}$ Autor correspondente: adrianapassos@pharma.ufrj.br (A.P. Oliveira)
} 
maio de 2006, que aprova a Política Nacional de Práticas Integrativas e Complementares (PNPIC) no Sistema Único de Saúde (SUS); a segunda foi o Decreto $\mathrm{n}^{\mathrm{0}}$ 5.813, de 22 de junho de 2006, que aprova a Política Nacional de Plantas Medicinais e Fitoterápicos (PNPMF) (1,2). Entre as ações, previstas no Programa da PNPMF $^{2}$, relativas ao acesso a plantas medicinais e fitoterápicos no SUS, destaca-se a elaboração da Relação Nacional de Plantas Medicinais de Interesse ao SUS (RENISUS) e a Relação Nacional de Plantas Medicinais e Fitoterápicos (RENAFITO).

O Ministério da Saúde visando estimular a geração de produtos, através da Portaria Ministerial MS/GM n 2.982 de 26 de novembro de 2009, aprovou o financiamento e execução para aquisição de derivados de algumas espécies vegetais, como a Mikania glomerata no elenco de Referência Nacional do Componente Básico da Assistência Farmacêutica (3). Em 2011, no âmbito da Fitoterapia, foi publicado o "Formulário de Fitoterápicos da Farmacopeia Brasileira" para dar suporte às práticas de manipulação e dispensação de fitoterápicos nos Programas de Fitoterapia no SUS, contemplando as normas de manipulação, oficializando as formulações para serem manipuladas de forma padronizada, como Cymbopogon citratus Stapf. (Capim-limão) na forma de preparação extemporânea (4). Os fitoterápicos do formulário também podem ser notificados na Anvisa, produzidos de acordo com a RDC no 13/2013 (5).

A Secretaria Municipal de Saúde de Volta Redonda (SMS/VR), através das Áreas Técnicas de Práticas Integrativas e Complementares e de Assistência Farmacêutica, ambas inseridas na Superintendência de Atenção e Vigilância em Saúde, desenvolve ações para implementar a Política Nacional de Prática Integrativas e Complementares (PNPIC) e a Política Nacional de Plantas Medicinais e Fitoterápicos no SUS. Portanto, em 2011, foi publicada a Lei Municipal n 4.832, que estabelece a Política Municipal de Práticas Integrativas e Complementares e possibilita a integração com a Política de Assistência Farmacêutica do município (6). Visando ampliar o trabalho na área de fitoterápicos, fez-se a estruturação do Arranjo Produtivo Local (APL), que engloba a cadeia de produção e sua integração na busca do desenvolvimento local, resgatando, valorizando, ampliando e qualificando o uso de plantas medicinais e dos fitoterápicos como elementos estratégicos de saúde.

\section{Materiais e Métodos}

O trabalho foi desenvolvido de maneira intersetorial e transversal, envolvendo a SMS/VR, gestora do projeto, e as instituições colaboradoras, de acordo com os respectivos eixos, A (cultivo das espécies medicinais), B (produção dos fitoterápicos), C (dispensação dos fitoterápicos), D (capacitação de recursos humanos) e E (manutenção dos colaboradores).

O eixo A representou o cultivo das espécies medicinais, englobando a identificação das localidades/áreas dos agricultores e das espécies vegetais, além da produção de mudas e a melhoria do horto.

Para identificação das localidades/áreas com histórico de cultivo orgânico e de sistemas de cultivo em larga escala de plantas medicinais na região do Médio Paraíba Fluminense, foi realizado o levantamento de dados nos sites oficiais do MAPA (Ministério da Agricultura Pesca e Abastecimento) e da EMATER-Rio (Empresa de Assistência Técnica e Extensão Rural do Estado do Rio de Janeiro). Além disso, foram realizados contatos diretos com os técnicos responsáveis do MAPA e da EMATER-Rio.

A identificação e a seleção dos agricultores interessados em participar de projeto de APL foram realizadas através de reuniões com agricultores associados pelo Sistema Participativo Garantido (SPG) com certificação de orgânico emitido pela Associação de 
Agricultores Biológicos do Estado do Rio de Janeiro (ABIO).

Para definição das espécies vegetais, foi realizado um levantamento pelos Agentes Comunitários de Saúde (ACS) junto à população sobre o uso de plantas medicinais em 15 Unidades Básicas de Saúde da Família (UBSF). Nessa seleção de espécies vegetais, foram considerados os seguintes fatores: espécies vegetais aclimatadas na região; áreas com histórico de cultivo e possibilidade de desenvolvimento socioeconômico; a prevalência de enfermidades passíveis de intervenção com o uso das espécies vegetais selecionadas.

A produção de mudas, a conservação e a disponibilização de mudas das espécies vegetais selecionadas para as lavouras a serem implantadas no âmbito do projeto foram realizadas em parceria com Instituto Federal do Rio de Janeiro (IFRJ) - Campus Pinheiral. Para tal, o viveiro de mudas do IFRJ trabalhou com a reprodução de material vegetal com identificação botânica assegurada. Foi utilizado o sistema convencional de produção de mudas associado com a micropropagação para garantir a manutenção das características genéticas do material e aumentar a velocidade da produção de mudas. A micropropagação foi realizada no laboratório de cultura de tecidos vegetais conforme $o$ protocolo padrão para cada espécie. Após o estabelecimento da cultura in vitro, as plantas foram transferidas para o solo e aclimatadas no viveiro de mudas para posterior plantio. A produção de mudas segue as normas que orientam a produção orgânica.

A melhoria da estrutura do horto para a produção de mudas do projeto APL na sede da cooperativa de produção e trabalho alternativo dos produtores e trabalhadores ligados à pastoral da criança dos municípios do médio Paraíba - COOP-PROALT, que visa cercar com tela a área de $5.000 \mathrm{~m}^{2}$ do horto, implantar um sistema de irrigação em toda a área, produzir composto orgânico e corrigir o solo.

O eixo B consiste na produção dos fitoterápicos por manipulação em Farmácia Viva (7). Para tal, a Farmácia Viva foi implantada em área pública do município (terreno da Fundação Beatriz Gama - FBG), localizado próximo ao centro de plantio e cultivo, e estruturado com recurso financeiro do BNDES. A área física foi planejada de acordo com a legislação vigente (8) para Farmácias Vivas, contendo os seguintes ambientes: área ou sala para as atividades administrativas; área ou sala de recebimento; áreas ou salas de processamento; área ou sala de armazenamento; área ou sala de controle de qualidade; sala ou local de pesagem de matérias-primas; sala de preparação; área de dispensação; vestiários; sala de paramentação; sanitários; área ou local para lavagem de utensílios e materiais de embalagem e depósito de material de limpeza. Além disso, houve a regularização junto ao órgão de vigilância sanitária local e o peticionamento da Autorização de Funcionamento de Empresa (AFE) junto à ANVISA.

O eixo C compreende a dispensação dos fitoterápicos, Mikania glomerata Spreng. (Guaco) e Cymbopogon citratus Stapf. (Capim-limão), de acordo com a Política Municipal de Assistência Farmacêutica (PMAF), através da Farmácia Municipal e dos dispensários existentes nas UBSF. Para tal, foi realizada a estruturação dos dispensários de fitoterápicos nas UBSF e a aquisição de recursos humanos.

A estruturação dos dispensários de fitoterápicos nas UBSF foi executada através da adequação das unidades aos padrões mínimos de armazenamento de medicamentos fitoterápicos. Além disso, foi implantado o Sistema Nacional de Gestão da Assistência Farmacêutica, denominado Hórus, nos dispensários das UBS beneficiadas pelo APL, visando à adequação dos serviços de informação para garantia da transmissão de dados.

A aquisição de recursos humanos dos dispensários de fitoterápicos foi efetuada através da contratação temporária ou terceirizada, compatíveis com as atividades a serem desempenhadas, permitindo à operacionalização dos dispensários na atenção básica, como o farmacêutico e os auxiliares de farmácia. 
O eixo D envolveu a capacitação dos profissionais envolvidos nas diferentes etapas da cadeia de produção e utilização de plantas medicinais e fitoterápicos e sobre o seu uso racional, qualificando os profissionais de nível médio e nível superior e os agricultores, além da elaboração de material informativo.

A capacitação de profissionais de nível médio atuantes nas UBSF foi realizada por meio de curso semipresencial com carga horária total de 54 horas em "Introdução ao Estudo de Plantas Medicinais e Fitoterápicos" promovido pelo Instituto Vital Brazil, sendo 12 horas presenciais, realizado em quatro meses com dois encontros presenciais de 6h, abordando os seguintes tópicos: a) aspectos históricos, de mercado, legais e éticos e conceitos aplicados; b) fitoquímica e farmacologia aplicadas; c) noções de farmacotécnica aplicadas; d) aspectos tradicionais em fitoterapia; e) aspectos de segurança e interações no uso de fitoterápicos e alimentos funcionais; f) orientação e preparação caseira de fitoterápicos e alimentos funcionais. A meta inicial foi capacitar 80 profissionais de nível médio, sendo 40 Agentes comunitários, 20 técnicos de enfermagem, 20 técnicos de saúde bucal.

A capacitação de profissionais de nível superior atuantes nas UBSF será realizada através de curso de pós-graduação na modalidade EAD, a ser promovido pela Associação Brasileira de Fitoterapia - ABFIT, com quatro encontros presenciais de $6 \mathrm{~h}$ cada durante 12 meses, abordando dois principais módulos, introdução a fitoterapia e farmacologia e clínica. A meta inicial foi capacitar 40 profissionais de nível superior, correspondendo ao total de 25 médicos, 10 cirurgiões dentistas, dois nutricionistas e três farmacêuticos para serem qualificados nos cuidados gerais com as plantas medicinais efitoterápicos, incluindo conhecimento sobre aspectos relacionados à manipulação e prescrição.

A capacitação de quatro profissionais agricultores das plantas medicinais escolhidas para o desenvolvimento do APL de Volta Redonda foi realizada visando à produção em sistema agroecológico. Para tal, a capacitação ocorreu por intermédio de atividades teóricas e práticas com visitas à área de cultivo das espécies, previstas a serem programadas duas vezes ao ano, durante dois anos.

A elaboração de material informativo foi realizada para divulgação de informações sobre plantas medicinais e fitoterápicos para os profissionais de saúde e os usuários do SUS. Dessa forma, foi realizada a confecção de material informativo impresso como folders, cartazes e cartilhas/manuais. A meta inicial estabelecida foi elaborar 60.000 folders e 2.500 cartazes, abordando os cuidados com plantas medicinais a serem distribuídos para a população através das UBSF. Além desses, foi planejado a elaboração de 500 cartilhas sobre orientação farmacêutica sobre preparação de remédios caseiros com plantas medicinais e 500 mementos terapêuticos das espécies vegetais selecionadas.

O eixo E engloba a manutenção das parcerias ou colaboradores através de articulação, interação e cooperação entre os agentes de toda cadeia de plantas medicinais e fitoterápicos. Portanto, a SMS/VR realizou parceria com outros níveis de governo e instituições, permitindo o desenvolvimento das ações com: 1) a Pastoral da Criança, através da COOP-PROALT para o beneficiamento primário das espécies vegetais; 2) Universidade Federal Fluminense, através do Laboratório de Tecnologia em Produtos Naturais (LTPN), para capacitação de profissionais de saúde; 3) Universidade Federal do Rio de Janeiro, através do desenvolvimento de formulações fitoterápicas; 4) Instituto Federal do Rio de Janeiro/Pinheiral, para assessoria técnica, capacitação de profissionais, controle de qualidade e armazenamento das mudas; 5) Instituto de Pesquisa Jardim Botânico do Rio de Janeiro, setor medicinal, para doação de espécies vegetais; 6) EMATER, para assessoria técnica; 7) Ministério de Agricultura Pesca e 
Abastecimento (MAPA), para fiscalização, assessoria técnica e capacitação profissional. 8) Herbário da Universidade Federal do Rio de Janeiro, departamento de Biologia para tombamento e determinação das exsicatas das espécies do projeto. Nesse contexto, foram realizadas oficinas de discussão visando à articulação e à interação, nas quais foram apresentados os avanços e desafios dos APL. Para tal, foi realizado o $1^{\circ}$ Fórum Regional de Promoção dos Arranjos Produtivos Locais da Região Médio Paraíba.

\section{Resultados}

A produção de plantas medicinais na cidade de Volta Redonda pela Cooperativa de Trabalho Alternativo dos Trabalhadores, vinculada à Pastoral da Criança do Estado do Rio de Janeiro Ltda. COOP-PROALT foi reconhecida pela EMATER-Rio. O MAPA também informou o cultivo de plantas medicinais nessa região pela COOP-PROALT, que apresenta histórico de cultivo orgânico e, portanto, constando no Cadastro Nacional de Produtores Orgânicos (9).

O MAPA e a Organização das Cooperativas Brasileiras - Estado do Rio de Janeiro (OCB/RJ) apresentaram a COOP-PROALT como a única de produção de plantas medicinais em pequena escala nessa região. Dessa forma, indicando a necessidade de construção futura nessa região de sistemas de produção em larga escala de plantas medicinais e fitoterápicos.

A definição das espécies vegetais foi realizada após o levantamento pelos Agentes Comunitários de Saúde (ACS) junto à população que apresentou o uso habitual de 27 espécies vegetais. Dentre as cinco mais frequentes, destacam-se Mikania glomerata (Guaco) e Cymbopogum citratus (Capim-Limão), que são espécies aclimatadas na região; apresentam áreas com histórico de cultivo e possibilidade de desenvolvimento socioeconômico; a prevalência de enfermidades passíveis de intervenção com o uso dessas drogas vegetais e medicamentos fitoterápicos, no caso doenças do aparelho respiratório e em saúde mental; a possibilidade de diminuição das principais causas de morbidade hospitalar. Portanto, o Guaco e Capim-Limão foram selecionados para o projeto de estruturação e implantação do APL-VR, que contempla a produção de droga vegetal seca nas apresentações de sachê contendo $3 \mathrm{~g}$.

De acordo com o estudo de Sombra (10), a escolha inicial dessas duas espécies vegetais é satisfatória do ponto de vista do uso tradicional, uma vez que os seus usos foram identificados em $66,66 \%$ e $33,33 \%$, respectivamente, dos 37 estudos etnobotânicos da região disponíveis na literatura, atualmente.

No ano de 2016, foram identificados e selecionados dois agricultores interessados em participar do projeto APL-VR durante a reunião com 24 agricultores associados pelo SPG com certificação de orgânico emitido pela ABIO. Os critérios adotados para seleção dos agricultores, além de apresentar a propriedade certificada como orgânica, possuir o talão do produtor ou Nota Fiscal, aceitar as recomendações da equipe do APL, comprometer-se a conservar e fazer a manutenção dos equipamentos e insumos fornecidos pela secretaria de saúde como contrapartida e, finalmente, vender a quantidade de drogas vegetais acordadas entre as partes. Quanto à área a ser cultivada, ficou definido o total de 100 a 200 metros quadrados para cada agricultor. A área definida a ser cultivada foi determinada pelos próprios agricultores levando em consideração a área que cada um possui. Em colaboração com o IFRJ-Pinheral, foi determinado o potencial produtivo através do estudo de viabilidade do cultivo das duas espécies vegetais selecionadas. As análises financeiras para implantação e manutenção de produção de guaco e capim limão foram estimados por intermédio dos custos de insumos, serviços, mão de obra e materiais. Após a análise econômica, foram estimados 
o cultivo do Guaco em espaldeira para obtenção de $1.650 \mathrm{Kg}$ de droga vegetal em 1 hectare e o cultivo de Capim-Limão para obtenção de $365 \mathrm{Kg}$ de droga vegetal em 1 hectare.

Em relação à produção de mudas, o Viveiro do IFRJ-Pinheral produziu 1.500 mudas de Guaco e 500 mudas de Capim-Limão, que foram disponibilizadas aos dois agricultores selecionados. Quanto à estratégia para obter 50.000 mil mudas, está diretamente ligada ao funcionamento do laboratório de tecidos localizado no IFRJCampus Pinheiral, que se encontra em fase de estruturação. Entretanto, espera-se, com a parceria APL-VR e IFRJ, gerar conhecimentos sobre a produção de mudas de plantas medicinais, contribuindo com o desenvolvimento do programa de plantas medicinais e fitoterápicos.

A melhoria do horto ainda se encontra em fase de aquisição dos itens de irrigação e da assistência técnica necessária. Além da produção e fonte de material genético, o horto é importante à pesquisa botânica, agronômica e química das plantas medicinais, servindo como centro de disseminação de conhecimentos.

Para a produção dos fitoterápicos, foi realizada a implantação da Farmácia Viva. Para tal, a área física dessa farmácia de manipulação foi dividida da seguinte forma: sala para as atividades administrativas $\left(7 \mathrm{~m}^{2}\right)$, sala de recebimento $\left(60 \mathrm{~m}^{2}\right)$, sala de processamento $\left(43 \mathrm{~m}^{2}\right)$, sala de armazenamento $\left(37 \mathrm{~m}^{2}\right)$, sala de controle de qualidade $\left(30 \mathrm{~m}^{2}\right)$, sala de preparação $\left(86 \mathrm{~m}^{2}\right)$, área de dispensação $\left(15 \mathrm{~m}^{2}\right)$, vestiário $\left(8 \mathrm{~m}^{2}\right)$, sala de paramentação $\left(8 \mathrm{~m}^{2}\right)$, e dois sanitários $\left(2 \mathrm{~m}^{2}\right.$ cada). Dessa forma, a área do laboratório de manipulação de fitoterápicos foi $100 \%$ estruturada conforme a legislação vigente. A adequação final do espaço encontra-se em fase de conclusão e acabamento, aguardando a autorização de funcionamento (AFE) da Vigilância Sanitária Local (VISA). Em seguida, as análises de controle de qualidade das matérias-primas e dos fitoterápicos serão realizadas juntamente com as Instituições Colaboradoras, IFRJ e UFRJ. As apresentações, na forma de sachê contendo $3 \mathrm{~g}$ de droga vegetal das espécies Guaco e Capim-Limão, serão preparadas de acordo com com o Formulário Nacional de Fitoterápicos. Estima-se a produção de 2.000 sachês de cada espécie citada, fabricados por ano.

Quanto à etapa de dispensação dos fitoterápicos, foi executada a estruturação dos dispensários nas UBSF através da implantação do sistema informatizado de dispensação Hórus em $100 \%$ das unidades. Além disso, houve a aquisição de recursos humanos para os dispensários de fitoterápicos por meio da contratação de um Farmacêutico e um auxiliar de farmácia, correspondente a $50 \%$ da meta inicial. Para tal, foi publicada a portaria $\mathrm{n}^{\circ}$ 017/2015 FMS/SMS, que atribui responsabilidade técnica da farmácia viva à servidora Aline Oliveira da Silva, matrícula 355739-PMVR e a contratação de um auxiliar de farmácia.

A capacitação de recursos humanos foi realizada para os profissionais de nível médio por intermédio da primeira turma do curso "Introdução ao Estudo de Plantas Medicinais e Fitoterápicos", finalizado no dia 17 de março de 2017, no qual foram capacitados 30 profissionais, representando, aproximadamente, $40 \%$ da meta. A segunda turma desse curso está prevista, para o início, em agosto de 2017. Em relação à capacitação de profissionais de nível superior, cinco profissionais médicos foram capacitados no curso a distância da Associação Médica Brasileira de Fitomedicina - SOBRAFITO, em parceria com o Ministério da Saúde, para médicos do SUS. Além desses, três Farmacêuticos fizeram curso de aperfeiçoamento em Fitoterapia em parceria com o LTPN da UFF.

Outros dois profissionais de nível superior, o coordenador do APL-VR e o responsável técnico pela Farmácia Municipal, são Especialistas em Gestão da Inovação em Fitomedicamentos (Farmanguinhos/FIOCRUZ) e também fizeram o curso "Orientação 
Técnica para Estruturação de Assistência Farmacêutica e Arranjo Produtivo Local em Plantas Medicinais e Fitoterápicos no Âmbito do SUS", conforme exigência do Edital (11) SCTIE/MS $\mathrm{n}^{0} 1 / 2014$, em que a SMS-VR foi contemplada com subsídios financeiros para estruturação do APL com sede no município de Volta Redonda (Região de Saúde Médio-Paraíba) do Estado do Rio de Janeiro.

As atividades de capacitação na área de agroecologia na região foram realizadas pelo IFRJ-Pinheiral (12), através de minicurso sobre manejo ecológico do solo na sede da COOP-PROALT para sócios da cooperativa, funcionários da Prefeitura Municipal de Volta Redonda e estudantes, no dia 5 de fevereiro de 2013. Em seguida, ocorreu o "Curso sobre produção de mudas de plantas medicinais, que inaugurou as ações do projeto - Desenvolvimento de Arranjos Produtivos Locais em VR" para 25 membros da COOP-PROALT, de Volta Redonda (13). Assim, foram promovidos: i) o aprendizado sobre a tecnologia de produção de plantas medicinais em larga escala; ii) a capacitação dos agricultores nos sistemas de cultivo orgânico; iii) a motivação de outros agricultores para investirem no cultivo orgânico de plantas medicinais no âmbito do APL. Além desses, na área de cultivo, foram capacitados 32 agricultores cooperados a COOPPROALT, com os cursos de aperfeiçoamento em "Agricultura Orgânica, Cultivo, Secagem e Armazenamento em Plantas Medicinais" e "Compostagem e Manejo de Resíduos Orgânicos", ambos realizados pelo MAPA/RJ.

No total, quatro profissionais agricultores participaram de outras capacitações, sendo um Curso de Produção de Mudas de Plantas Medicinais, realizado no IFRJ, sob a Coordenação do professor Marcelo Santos de Souza e uma Visita Técnica ao APL Petrópolis, pela Fiocruz-Palácio do Itaboraí.

Em relação à divulgação por intermédio de material informativo, foi elaborado e impresso o total de 500 cartazes de "Plantas Medicinais mais utilizadas em Volta Redonda/RJ". Atualmente, o fôlder "Plantas tóxicas", a cartilha dos usuários e o memento terapêutico estão em fase de seleção do conteúdo, revisão e finalização.

Quanto à manutenção dos colaboradores, foram realizadas oficinas de discussão para abordar os principais desafios e apontar as melhorias. Em 2010, com objetivo de sensibilizar e incluir os profissionais da rede pública de saúde na discussão das estratégias para implantação dessas práticas, foi realizado o "I Fórum Municipal de Práticas Integrativas e Complementares no SUS", do qual participaram 200 profissionais. Em relação à Fitoterapia, dois temas foram debatidos: "Como Melhorar a Saúde com Plantas Medicinais" e "Legislação de Farmácia Viva". Esse Fórum promoveu o interesse em capacitar os profissionais da rede, a percepção de lideranças locais sobre a importância dessa Política e ampliou o debate para o legislativo municipal.

Em de 31 de agosto de 2016, foi realizado o $1^{\circ}$ Fórum Regional de Promoção dos Arranjos Produtivos Locais da Região Médio Paraíba com a participação de cinco palestrantes convidados, profissionais ligados às instituições parceiras do projeto como o Instituto Vital Brazil, a UFF, a FIOCRUZ, o MAPA e o IFRJ, atingindo a meta de $100 \%$ de adesão das vagas disponíveis.

\section{Discussão}

O Ministério da Saúde, desde 2012, financia projetos de beneficiamento de Arranjo Produtivo Local (APL), que se caracteriza como sendo "aglomerações de empreendimentos de um mesmo ramo, localizados em um mesmo território, que mantêm algum nível de articulação, interação, cooperação e aprendizagem entre si e com os demais atores locais (governo, pesquisa, ensino, instituições de crédito" (14-15). 
Em 2014, a Secretaria Municipal de Saúde de Volta Redonda foi contemplada com subsídios financeiros do Ministério da Saúde (16) para estruturação do APL-Volta Redonda (Região de Saúde Médio-Paraíba) do Estado do Rio de Janeiro.

O APL-Volta Redonda contempla a produção de droga vegetal das espécies Mikania glomerata e Cymbopogum citratus nas apresentações droga vegetal seca $3 \mathrm{~g}$ sachê. De acordo com Sombra (10), a escolha inicial dessas duas espécies vegetais é satisfatória do ponto de vista do uso tradicional da Região de Médio-Paraíba e a sustentabilidade do APL vai depender de mudanças no perfil dos serviços relacionados à Fitoterapia, ofertados nos municípios da Região de Saúde Médio-Paraíba (dimensão social), associado à garantia do retorno financeiro aos atores que representam o início da cadeia produtiva do APL, nesse caso, a COOP-PROALT (dimensão econômica).

Atualmente, a Região Médio-Paraíba não possui a dispensação de fitoterápicos manipulados, mas apenas de fitoterápicos industrializados, que são adquiridos com recursos do componente básico da assistência farmacêutica, o qual cresceu $25 \%$ na região do APL (10). Conforme descrito por Michiles (17), os serviços de fitoterapia junto aos municípios do Estado do Rio de Janeiro apresentam questões comuns à maioria dos municípios do país, como a necessidade de maior comprometimento dos gestores da área de saúde. Gomes (18) destacou a importância de identificar os parceiros institucionais para realização de trabalho conjunto para garantia da manutenção dos Serviços de Fitoterapia implantados nos municípios do Estado do Rio de Janeiro. Além disso, a escolha dos fitoterápicos a serem produzidos é também essencial para o fortalecimento do APL ou para um bom direcionamento para as atividades a serem desenvolvidas nesse arranjo.

O APL-Volta Redonda de plantas medicinais e fitoterápicos apresentou algumas características principais como territorialização, articulação de atores sociais, integração de serviços, troca de saberes/conhecimentos/tecnologias e instituição de formas inovadoras de organização. Esses princípios estão harmonizados com o SUS, de descentralização, regionalização e organização dos serviços em rede, possibilitando uma maior disponibilização e utilização de recursos para a promoção, prevenção e recuperação da saúde (19).

\section{Considerações finais}

Selecionado por meio do Edital SCTIE/MS n ${ }^{\circ} 1 / 2014$ (11), conforme as diretrizes da Política e do Programa Nacional de Plantas Medicinais e Fitoterápicos, o projeto APL de Volta Redonda foi desenvolvido e estruturado, visando garantir à população usuária do SUS Volta Redonda o acesso seguro e o uso racional de plantas medicinais e fitoterápicos, promovendo o uso sustentável da biodiversidade e o desenvolvimento da cadeia produtiva, que resulta em desenvolvimento econômico, tecnológico e social.

\section{Referências}

1. Brasil. Ministério da Saúde. Portaria $n^{\circ} .971$ de 03 de maio de 2006. Aprova a Política Nacional de Práticas Integrativas e Complementares (PNPIC) no Sistema Único de Saúde. Diário Oficial da União 2006; 04 mai.

2. Brasil. Presidência da República. Decreto n 5813 de 22 de junho de 2006. Aprova a Política Nacional de Plantas Medicinais e Fitoterápicos e dá outras providências. Diário Oficial da União, 2006; 23 jun.

3. Brasil. Ministério da Saúde. Portaria n 2982 de 26 de novembro de 2009. Aprova as normas de execução e de financiamento da Assistência Farmacêutica na Atenção Básica. Diário Oficial da União 2009; 26 nov.

4. Brasil. Agência Nacional de Vigilância Sanitária. Formulário de Fitoterápicos da Farmacopéia 
Brasileira. Diário Oficial da União 2011; 11 nov.

5. Brasil. Ministério da Saúde. Resolução. RDC no 13 de 14 de março de 2013. Dispõe sobre as Boas Práticas de Fabricação de Produtos Tradicionais Fitoterápicos. Diário Oficial da União, 2013; 15 mar.

6. Prefeitura Municipal de Volta Redonda. Política Municipal de Práticas Integrativas e Complementares no Município de Volta Redonda, RJ. [acessado 2017 jul 10]. Disponível em: http://www.portalvr.com/images/leis_municipais_2011/LEI\%20MUNICIPAL\%204.832.pdf.

7. Brasil. Ministério da Saúde. Resolução. Portaria no 886 de 20 de abril de 2010. Institui a Farmácia Viva no âmbito do Sistema Único de Saúde (SUS). Diário Oficial da União, 2010; 22 abr.

8. Brasil. Ministério da Saúde. Resolução. RDC no 18 de 03 de abril de 2013. Dispõe sobre as boas práticas de processamento e armazenamento de plantas medicinais, preparação e dispensação de produtos magistrais e oficinais de plantas medicinais e fitoterápicos em farmácias vivas no âmbito do Sistema Único de Saúde (SUS). Diário Oficial da União, 2013; 04 abr.

9. Brasil. Ministério da Agricultura, Pecuária e Abastecimento. Cadastro Nacional de Produtores Orgânicos. $\quad$ [acessado jul 2017 10]. Disponível em: http://www.agricultura.gov.br/assuntos/sustentabilidade/organicos/cadastro-nacional-produtoresorganicos.

10. Sombra AC. APL-Volta Redonda, uma abordagem regional dos aspectos etnobotânicos, agroecológicos e serviços relacionados à fitoterapia [monografia especialização]. Rio de Janeiro. Instituto de Tecnologia em Fármacos, Farmanguinhos; 2016.

11. Brasil. Ministério da Saúde. Secretaria de Ciência, Tecnologia e Insumos Estratégicos. Edital $n^{\circ} 1$, de 30 de maio de 2014. Seleção de Projetos de Estruturação e Consolidação de Assistência Farmacêutica em Plantas Medicinais e Fitoterápicos. Diário Oficial da União, Brasília, DF, 2 de junho de 2014. Seção 3, p. 141.

12. Instituto Federal do Rio de Janeiro. Informativo Campus Nilo Peçanha - Pinheiral. Ano 4 - n 36 Março 2013. [acessado 2017 jul 10]. Disponível em: http://www.ifrj.edu.br/webfm_send/4924.

13. Instituto Federal do Rio de Janeiro. Informativo Campus Nilo Peçanha - Pinheiral. Ano 6 - $\mathrm{n}^{\mathrm{o}} 47$ Março 2015. [acessado 2017 jul 10]. Disponível em: http://www.ifrj.edu.br/sites/default/files/webfm/images/Informativo\%20IFRJ\%20CANP\%20Edi\%C3 $\%$ A7\% $3 \%$ A3o\%2047.pdf.

14. Brasil. Portaria Interministerial $n^{\circ} 2.960$ de 9 de dezembro de 2008. Aprova o Programa Nacional de Plantas Medicinais e Fitoterápicos e cria o Comitê Nacional de Plantas Medicinais e Fitoterápicos. Diário Oficial da União, 2008; 10 dez.

15. Brasil. Ministério da Saúde. Secretaria de Ciência, Tecnologia e Insumos Estratégicos. Edital $n^{\circ} 1$, de 26 de abril de 2012. Seleção de propostas de Arranjos Produtivos Locais no âmbito do SUS, conforme a política e o programa nacional de plantas medicinais e fitoterápicos. Diário Oficial da União, Brasília, DF, 27 abril 2012. Seção 3, p. 145.

16. Brasil. Ministério da Saúde. Secretaria de Ciência, Tecnologia e Insumos Estratégicos. Portaria $n^{\circ}$ 2.323, de 23 de outubro de 2014. Aprova o repasse dos recursos de investimento e custeio, em parcela única para os Municípios e Estados selecionados pelo Edital $n^{\circ}$ 1/SCTIE/MS, de 30 de maio de 2014.

17. Michiles E. Diagnóstico situacional dos serviços de fitoterapia no Estado do Rio de Janeiro. Rev Bras Farmacogn, 2004; 14 (Supl.0): 16-19.

18. Gomes AMO. Diagnóstico para implantação do programa nacional de plantas medicinais e fitoterápicos, nos municípios do Estado do Rio de Janeiro [monografia especialização]. Rio de Janeiro. Instituto de Tecnologia em Fármacos, Farmanguinhos; 2012.

19. Torres KR. Os arranjos produtivos locais (APLs) no contexto da implementação da Política e do Programa de Plantas Medicinais e Fitoterápicos [dissertação]. Rio de Janeiro. (RJ). Escola Nacional de Saúde Pública Sergio Arouca da Fiocruz; 2013. 